\title{
Successful application of human-based methyl capture sequencing for methylome analysis in non-human primate models
}

Ja-Rang Lee ${ }^{1 \dagger}$, Dong-Sung Ryu ${ }^{2 \dagger}$, Sang-Je Park ${ }^{3 \dagger}$, Se-Hee Choe ${ }^{3,5}$, Hyeon-Mu Cho ${ }^{3,5}$, Sang-Rae Lee ${ }^{3,5}$, Sun-Uk Kim ${ }^{4,5}$, Young-Hyun $\mathrm{Kim}^{3,5^{*}}$ and Jae-Won Huh ${ }^{3,5^{*}}$ (D)

\begin{abstract}
Background: The characterization of genomic or epigenomic variation in human and animal models could provide important insight into pathophysiological mechanisms of various diseases, and lead to new developments in disease diagnosis and clinical intervention. The African green monkey (AGM; Chlorocebus aethiops) and cynomolgus monkey (CM; Macaca fascicularis) have long been considered important animal models in biomedical research. However, non-human primate-specific methods applicable to epigenomic analyses in AGM and CM are lacking. The recent development of methyl-capture sequencing (MC-seq) has an unprecedented advantage of cost-effectiveness, and further allows for extending the methylome coverage compared to conventional sequencing approaches.

Results: Here, we used a human probe-designed MC-seq method to assay DNA methylation in DNA obtained from 13 CM and three AGM blood samples. To effectively adapt the human probe-designed target region for methylome analysis in non-human primates, we redefined the target regions, focusing on regulatory regions and intragenic regions with consideration of interspecific sequence homology and promoter region variation. Methyl-capture efficiency was controlled by the sequence identity between the captured probes based on the human reference genome and the AGM and CM genome sequences, respectively. Using reasonable guidelines, 56 and $62 \%$ of the human-based capture probes could be effectively mapped for DNA methylome profiling in the AGM and CM genome, respectively, according to numeric global statistics. In particular, our method could cover up to 89 and $87 \%$ of the regulatory regions of the AGM and CM genome, respectively.
\end{abstract}

Conclusions: Use of human-based MC-seq methods provides an attractive, cost-effective approach for the methylome profiling of non-human primates at the single-base resolution level.

Keywords: Methyl-capture sequencing, DNA methylation, Animal model, Cynomolgus monkey, African green monkey

\section{Background}

Because of their close evolutionary relationship with humans, non-human primates (NHPs) are considered valuable animal models for biomedical research [1]. NHPs show a high degree of similarity to humans in the genome sequence; e.g., 98.77\% similarity with chimpanzee [2], 93. $5 \%$ similarity with rhesus monkey [3], and $92.83 \%$ similarity with cynomolgus monkey [4]. In addition, NHPs share many physiological, immunological, and morphological

\footnotetext{
* Correspondence: kyh@kribb.re.kr; huhjw@kribb.re.kr

${ }^{\dagger}$ Equal contributors

${ }^{3}$ National Primate Research Center, Korea Research Institute of Bioscience and Biotechnology, Cheongju 28116, Republic of Korea

Full list of author information is available at the end of the article
}

similarities with humans. Moreover, they have numerous advantages as animal models for translation to humans, including controllability of environmental factors, ease of scale, and comparability of results [5]. Therefore, use of an NHP animal model could provide particularly valuable information in the development of vaccines and drugs, and for establishing preventive and therapeutic measures against emerging pathogens [6].

Among the NHPs, crab-eating or cynomolgus macaque (CM; Macaca fascicularis), rhesus macaque (Macaca mulatta), and African green monkey (AGM; Chlorocebus aethiops) are most commonly used for biomedical research [7]. These primates belong to the group of Old

(c) The Author(s). 2018 Open Access This article is distributed under the terms of the Creative Commons Attribution 4.0 International License (http://creativecommons.org/licenses/by/4.0/), which permits unrestricted use, distribution, and 
World monkeys, and diverged from the common ancestor of human and Old World monkeys about 32 million years ago [8]. Their close relationship to humans has made these primate species particularly suitable as animal models for biomedical research and evolutionary studies [9-12]. Rhesus macaques, of Indian origin, have served as a traditional animal model for human diseases [1]. However, since the export of rhesus macaques from India was banned in 1978, they have become harder to obtain. As an alternative, CM has been more widely adopted as an animal model for human disease. In addition, CM has several important advantages as an animal model compared to rhesus macaque: (1) easy handling due to its smaller body size and weight; (2) low cost and better availability for experimental use; and (3) lack of seasonal fertility [13]. AGM has long been considered an important animal model for biomedical applications such as in human immunodeficiency virus research, because they show resistance to simian immunodeficiency virus [5]. Recently, the draft genomes of AGM and CM were published, and the sequences are now available in various genomic databases (AGM GenBank Assembly ID, GCA_000409795.2; CM GenBank Assembly ID, GCA_000364345.1). Therefore, these primates could now serve as attractive animal models, and their contribution to biomedical research is expected to increase in the coming years.

DNA methylation, as an important epigenetic regulation, occurs at the 5-carbon residues of cytosine via the addition of a methyl group, which is catalyzed by DNA methyltransferases. In mammalian genomes, DNA methylation is predominantly found in CpG dinucleotides. In particular, methyl-cytosine is observed in up to $80 \%$ of normal human cells [14]. However, the occurrence of methylation is generally suppressed in GC-rich DNA, consisting of several regions known as $\mathrm{CpG}$ islands (CGIs). Approximately $60 \%$ of all known human genes are associated with CGIs in their promoter regions [15]. Methylation in the promoter region is closely associated with downstream gene silencing, and this modification not only regulates gene expression but also plays a role in numerous cellular processes, including X-chromosome inactivation, imprinting, embryonic development, maintenance of genomic stability, and transposon inactivation [16]. In somatic cells, DNA methylation patterns are stably maintained, and are inherited to daughter cells through mitotic cell division. However, they are not permanent. In fact, changes in DNA methylation are dynamically regulated during the mammalian life cycle [17]. In addition, changes in DNA methylation patterns are induced by several extrinsic factors derived from environmental exposure, ranging from a natural physiological response to environmental changes to those associated with the development of diseases such as neurodegenerative disorders, diabetes, cardiovascular disease, and various types of cancer [18]. Therefore, an aberrant DNA methylation change is a highly promising molecular biomarker for the early detection, diagnosis, and prognosis of complex or chronic diseases. For this reason, it is of great value to investigate the DNA methylome of AGM and $\mathrm{CM}$ as important animal models for human disease. However, establishment of a genome-wide approach to explore the DNA methylome of NHPs has thus far been hampered by the lack of suitable tools and cost limitations.

Many methods for genome-wide DNA methylation analysis at the single-base resolution are available for human samples, which can be divided into two main categories: microarray- and next-generation sequencing (NGS)-based methods. The microarray-based Infinium Human Methylation450 BeadChip Array (Infinium $450 \mathrm{~K}$ ) has been widely used for epigenetics analyses owing to its advantages of cost-effectiveness, rapid sample processing time, and possibility for highthroughput processing of bulk samples [19]. However, the main limitation of microarray-based methods is the requirement for a fixed number of probes that target specific genome loci. Therefore, microarraybased methods are only suitable for screening a genome at known methylation-altered loci. Alternatively, NGS-based methods can be further refined according to the targeted genome regions. Whole-genome bisulfite sequencing (WGBS) is considered the gold-standard method, which can provide the highest genomic coverage and nucleotide resolution for quantification of DNA methylation [20]. However, this method is associated with substantial costs and a relatively long processing time for obtaining highquality sequences, which have limited its widespread application. To reduce the associated sequencing costs and processing time, methyl-capture sequencing (MC-seq) is an attractive option, which allows for the selection of predefined genomic regions, and utilizes target-specific genomic loci of physiological and clinical interest [21]. The MC-seq method has various advantages of costeffectiveness, broader genome coverage, and avoidance of the bias due to CpG-rich repeats. However, before the MC-seq method can be applied to NHPs, it is essential to first determine the applicability of the human genomebased captured probes for these models.

Toward this end, in this study, we sought to determine the applicability and accuracy of a human-based MC-seq kit to the AGM and CM genomes. We redefined the MC-seq target region for methylome analysis considering the probe sequence similarity and variation in the promoter regions of the same genes between human and NHPs. Adaptation of the 
established human MC-seq method for NHPs can be a powerful tool for epigenome analysis, and help provide novel information about DNA methylation alteration patterns with direct clinical translation.

\section{Methods}

\section{Sample collection and extraction of primate genomic DNA samples}

Ethical approval for collecting blood samples of cynomolgus macaques and African green monkeys was granted by the Institutional Animal Care and Use Committee (KRIBB-AEC-140007, KRIBB-AEC-15031 \& KRIBB-AEC-15046) of the Korea Research Institute of Bioscience and Biotechnology (KRIBB). Animal preparation and study design were conducted according to the Guidelines of the Institutional Animal Care and Use Committee. Blood samples of cynomolgus macaques and African green monkeys were provided by the National Primate Research Center of Republic of Korea.

Genomic DNA samples were isolated from the peripheral blood of 13 specific pathogen-free female CMs (1-9 years old), one female AGM (20 years old), and one male AGM (16 years old), which were collected in each of the last 2 years for periodic health monitoring. Blood samples were collected by venipuncture and stored in PAXgene tubes (PreAnalytiX, Hombrechtikon, Switzerland). Genomic DNA was extracted using the PAXgene Blood DNA Kit (Qiagen, Hilden, Germany).

\section{Definition of targeted genomic regions}

Targeted genomic regions were divided into regulatory and intragenic regions (Fig. 1). Regulatory regions contain promoters, CGIs, and CGI flanking regions (shore and shelf). As one of the most important regulatory regions, CGI regions were predicted by cpgreport, a widely used CGI prediction tool in the EMBOSS package, using default parameters [22]. The shore and shelf flanking regions were determined from the predicted CGIs, which span up to $2 \mathrm{~kb}$ from the end or start of the CGI and $\geq 2 \mathrm{~kb}$ from the end or start of the shore, respectively (Fig. 1) [23, 24]. In addition, promoter regions were defined to $2 \mathrm{~kb}$ upstream from the transcription start site (TSS) in present study. To determine the span of the promoter region, the TSS was calculated as the start site of the longest transcript among the transcripts associated with the same gene symbol. Ensembl 75 was used to calculate the promoter region and to define intergenic or intragenic genomic regions.

\section{Homologous probe region (HPR)}

The NHP genome has a high level of sequence similarity to the human genome in view of the close evolutionary relationship. To identify the NHP target region, we extracted the human genome sequences located on the captured region by the probes of the SureSelect ${ }^{\mathrm{XT}}$ Human Methyl-Seq (Agilent Technologies, Santa Clara, CA, USA). The extracted genome sequences were aligned to the NHP genome by the local alignment tool BLAT [25], which is particularly
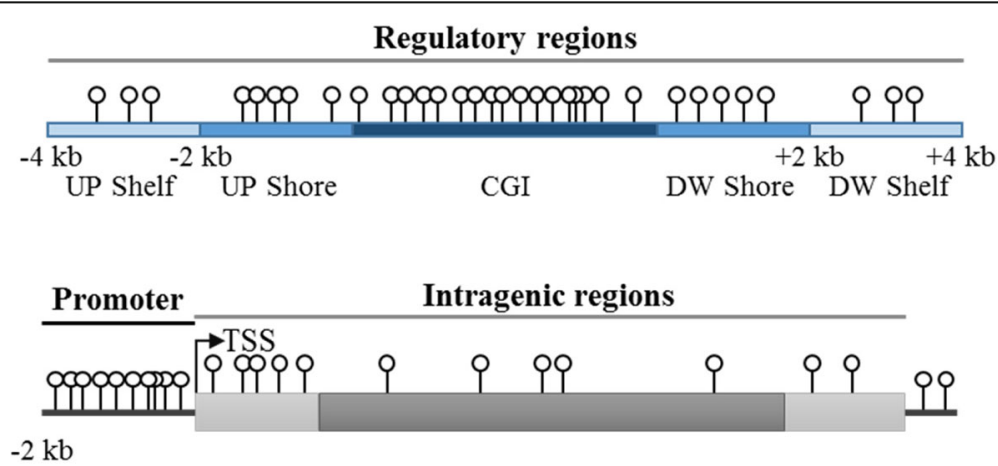

$$
\begin{aligned}
& \square \text { Shelf } \square \text { Shore } \square \text { CGI } \\
& \text { Exon } \square \text { CDS } 9 \text { CG site }
\end{aligned}
$$

Fig. 1 Definitions of various genomic regions used for methyl-capture sequencing in this study. Targeted genomic regions can be separated into regulatory regions and intragenic regions. Regulatory regions included CG islands (CGIs) with the surrounding CGI shore, CGI shelf, and promoter region. Intragenic regions are organized into all exon regions, including the coding sequence (CDS), excluding introns. US, upstream; DS, downstream; TSS, transcription start site 
useful to align consecutive genomic sequences as much as possible. For blat parameters, we used default values which just consider DNA alignment $(-\mathrm{q}$ $=$ dna; - out $=$ blast $8 ;-t=d n a)$. Then, we selected the sequences based on the alignment options (identity and e-value) among the various aligned regions. The alignment results are summarized in Additional file 1: Table S1.

The homologous probe region has to be determined to allow for the efficient and precise use of the target genomic region with sufficient average sequence depth for confidence. In this study, we selected an identity value of $85 \%$ and an e-value of 1 . $0 \times 10^{-10}$, which allowed for the target region to reach a near-average depth of 30 -fold for each CpG site, which is considered to be a reasonable depth for methylation analysis [26]. These values also permit sufficient use of the coverage in the SureSelect ${ }^{\mathrm{XT}}$ Human Methyl-Seq $(84 \mathrm{Mb})$ up to $\sim 60 \%$. We redefined the calculated target region determined using this approach as the HPR.

\section{Orthologous promoter region (OPR)}

In general, it is important to estimate the methylation level of $\mathrm{CpG}$ sites located on the promoter region from the perspective of gene regulation. Since probes of the human toolkit are designed to capture portions of the promoter regions, the whole promoter regions annotated in the Ensembl or UCSC databases, which are generally used for methylome analysis, are not targeted. Therefore, to compensate for this partial annotation and achieve a more expanded analysis of the uncovered regions that are not included by the sequence homology-based method, we added the OPR to the redefined target regions (Fig. 2). To define the captured gene symbols by the human tool kit, we listed the gene symbols that overlapped by more than $60 \%$ with the human targeted probe region. We then selected the gene symbols that matched with NHP gene symbols, and the NHP promoter regions were re-calculated to $2 \mathrm{~kb}$ in the $5^{\prime}$ direction from the TSS.

\section{Redefined target region}

The CG distribution of the redefined target region corresponding to regulatory and intragenic regions is summarized in Additional file 2: Table S2. The redefined target region includes 1,680,406 (HPR, 1.66 million) and 1,812,429 (HPR, 1.81 million) CG sites in AGM and CM genome, and could cover 53.5\% (HPR, 52.8\%) and 57.7\% (HPR, 57.6\%) of the CG sites compared to human targeted CG sites, respectively. In this study, we redefined the new target region focusing on the sequence homology between the NHP and human genomes. To consider a more extended HPR, we could adjust the mismatch parameters to be more loose during the alignment (Additional file 1: Table S1).

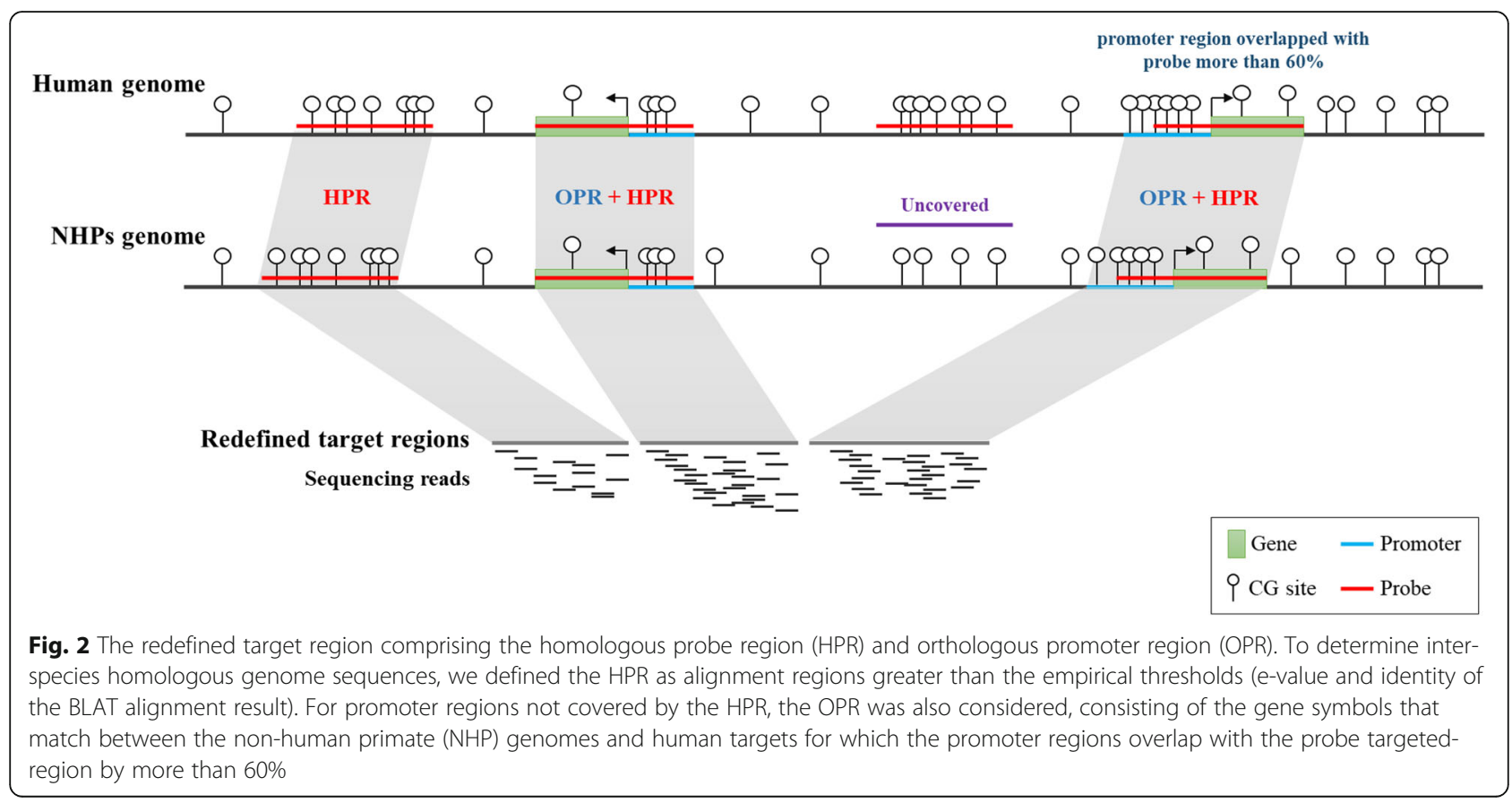




\section{MC-seq and analysis}

For NHP MC-seq analysis, DNA extracted from blood samples of three AGMs and 13 CMs were sequenced. We prepared the genomic libraries using the SureSelect ${ }^{\mathrm{XT}}$ Methyl-Seq Target Enrichment System [27] for NHP MC-seq. The probes of this human toolkit are designed to capture 3.7 million CpG sites over an 84-Mb region, targeting DNA fragments of CG-rich regions (CGIs, including the shore and shelf), promoter regions, as well as known cancer- and tissue-specific differentially methylated regions (DMRs). All NHP samples were sequenced using the same workflow. In brief, genomic DNA was randomly sheared and then DNA fragments of 150-200 bp were extracted. The DNA fragments were subjected to end repair, adapter ligation, hybridization to SureSelect ${ }^{\mathrm{XT}}$ Methyl-seq Capture Library, streptavidin bead enrichment, bisulfite conversion, and PCR amplification, and then unique index tags were added by PCR amplification. DNA sample libraries were sequenced with an Illumina Hiseq2000 sequencer according to the manufacturer's instructions. The length of the sequenced read was 101 base pair-ends. For mapping of the sequenced reads, we used the reference sequences GCA_000409795.2 and GCA_000364345.1 for AGM and $\mathrm{CM}$ with the Ensembl 78 and Ensembl preversion annotation databases, respectively. In the case of the CM reference, the Ensembl and NCBI databases could not provide sufficient annotation information for methylome analysis, since this reference is a pre-assembled version. The human reference genome sequence hg19 in the UCSC database was used for comparison or analysis with the Ensembl 75 annotation database. For mapping of bisulfite-converted reads, we used Bismark [28], which provides the minimized bias result by using a best-hit alignment strategy. In order to improve the accuracy of bisulfite alignment, we designated -N parameter as 0 (maximal mismatches permitted). At the case of other parameters, default values were used. The same version of the Bismark package was used for uniquely mapped sequences to the reference, de-duplication, and cytosine calling.

\section{Results}

Redefined target region for MC-seq analysis using a human probe capture system in the AGM and CM genomes

To employ the human methyl-captured toolkit for NHPs, we searched the AGM and CM genome sequences with the human capture probe sequences using BLAT with an identity cut-off of $85 \%$ and an e-value threshold of $1.0 \times$ $10^{-10}$. Overall, 56.3 and $61.9 \%$ of all human capture probes could be successfully mapped to the AGM and
CM genome, respectively (Fig. 3a, b; see Additional file 1: Table S1 for details). To further investigate how the redefined target regions are constructed on each annotated genomic region, we analyzed the CG site distribution according to the annotated genomic region (Fig. 3c). For comparison with AGM and CM, we also calculated the CG site distribution of the human toolkit on the human genomic region, respectively. The redefined regions covered 349,499 (HPR, 326,734) and 309,205 (HPR, 305,570) CG sites of promoter regions in AGM and CM (Additional file 2: Table S2). This coverage shows that the human target region overlapped with 67.8 and $58.1 \%$ of regions in the AGM and CM genome, respectively. Another encouraging fact was that the redefined regions could cover 752,032 (HPR, 747,884) and 798,668 (HPR, $796,929)$ of the CGI regions in AGM and CM (Additional file 2: Table S2). This extent of coverage corresponds to 88.6 and $86.5 \%$ of the coverage for the human targeted CGI regions. The additional OPR expanded 22,765 and 3635 CG sites in total for the targeted regions in AGM and $\mathrm{CM}$, respectively (Additional file 2: Table S2). The OPR for CM could not be extended or covered more than that of AGM since the CM annotation database (preversion) is not as well established as the AGM annotation database with respect to gene symbols. Thus, addition of the OPR appears to be more effective when dealing with well-established genomes; nevertheless, use of the OPR allowed for more expanded analysis of the uncovered regions in both the AGM and CM genomes.

\section{Evaluation of human-based MC-seq performance for AGM and $\mathrm{CM}$ samples}

The DNA methylomes of AGM and CM samples were generated using MC-seq with a SureSelect capture system and bisulfite-conversion approach. The mapping statistics are summarized in Fig. 4a and Additional file 3: Table S3. On average, 82 million pair-end reads were generated per sample, 62 million of which aligned uniquely to the bisulfite-converted AGM and CM genome. We sequenced each sample up to nearly 100 -fold as a goal to acquire a sufficient amount of reads (more than 40-fold depth after de-duplication) that could then be used in the methylation-level calling. In the case of samples A03 and C10, we conducted additional sequencing to satisfy our criteria to acquire a sufficient amount of de-duplicated reads. The numbers of mapped reads between samples relative to the corresponding reference were similar, and the ratio of mapped reads was greater than $70 \%$. After removing multiply mapped reads on the genome, the ratio of uniquely mapped reads in most of the samples was also greater than $70 \%$, indicating that the sequencing data was of good quality. The protocol for the MC-seq method might be accompanied by a high level of 

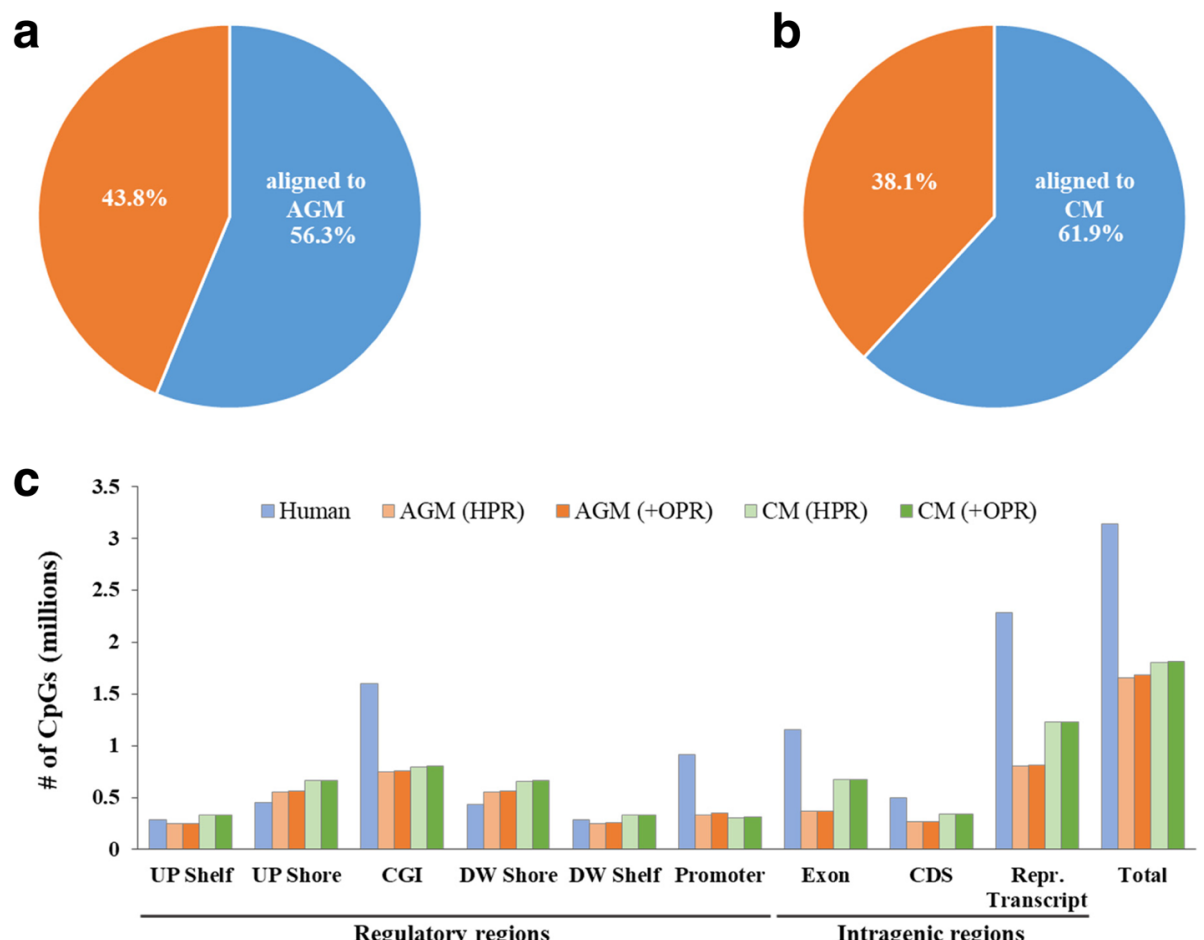

Fig. $3 \mathrm{CpG}$ content distribution according to each genomic region in redefined targeted regions. The pie charts show the percentages of sequences identified with the SureSelect human toolkit that could be aligned to the a African green monkey (AGM) and $\mathbf{b}$ cynomolgus macaque (CM) genome with an identity threshold of $85 \%$ and an e-value $<\mathrm{e}^{-10}$. $\mathbf{c}$ The height of bar graphs represents the number of CpGs covered by the homologous promoter region (HPR) and orthologous promoter region (OPR) methods

duplicated reads caused by doubling of the PCR amplification. Therefore, we overcame this problem by adopting an acquisition strategy for de-duplicated reads. The average proportion of duplicated read was $25 \%$ for the primate genomes. Finally, we could secure the deduplicated reads from more than 39 million reads of each sample, which could then be used for actual cytosine calling analysis (Additional file 3: Table S3).

After the alignment process, we filtered out the only on-targeted reads using the redefined target region as a guide. The ratio of on-targeted reads compared with de-duplicated reads ranged from 50.6 to $62.1 \%$. The average ratio of on-targeted reads for the HPR and for the HPR plus OPR analysis was 59.8 and $59.9 \%$, respectively. The average depths for the targeted regions with their cumulative percentages of CG sites according to depth in the target region are summarized in Additional file 4: Table S4. The cytosine calling depths were greater than 40 -fold in all cases, except for sample A03. Furthermore, more than $90 \%$ of the targeted CG sites were covered at $a \geq 5$ fold calling depth (Fig. 4b). These statistics show that our target region could provide adequate detailed resolution and capture performance at the on-targeted region to effectively estimate the methylation level using the human toolkit. If we set a more extended
OPR with a loose overlap ratio of gene symbols using the human toolkit, we could obtain a greater number of on-targeted reads; however, this would come at a cost of low depth coverage of on-target reads. Finally, based on our criteria for a redefined target region, the comprehensive distribution maps of the target regions in the AGM and CM genomes were obtained (Fig. 5).

\section{Characterization of methylation levels in AGM and CM models}

To confirm the accurate detection of methylome status using the human toolkit for analysis of AGM and $\mathrm{CM}$ samples, we investigated methylation levels with various genomic regions based on our MC-seq data (Fig. 6). Additional file 5: Table S5 shows the average methylation levels with their standard deviations for each genomic region. For CG methylation level estimation, we merged each strand of DNA. In the mammalian genome, intergenic DNA, exon regions, and transposable element sequences commonly show high methyl-cytosine levels [29], whereas the promoter and CGI regions are usually hypomethylated compared with the intragenic regions. Furthermore, CGIs and CGI flanking regions (including the shore and shelf) have been reported to show gradual hypermethylation 

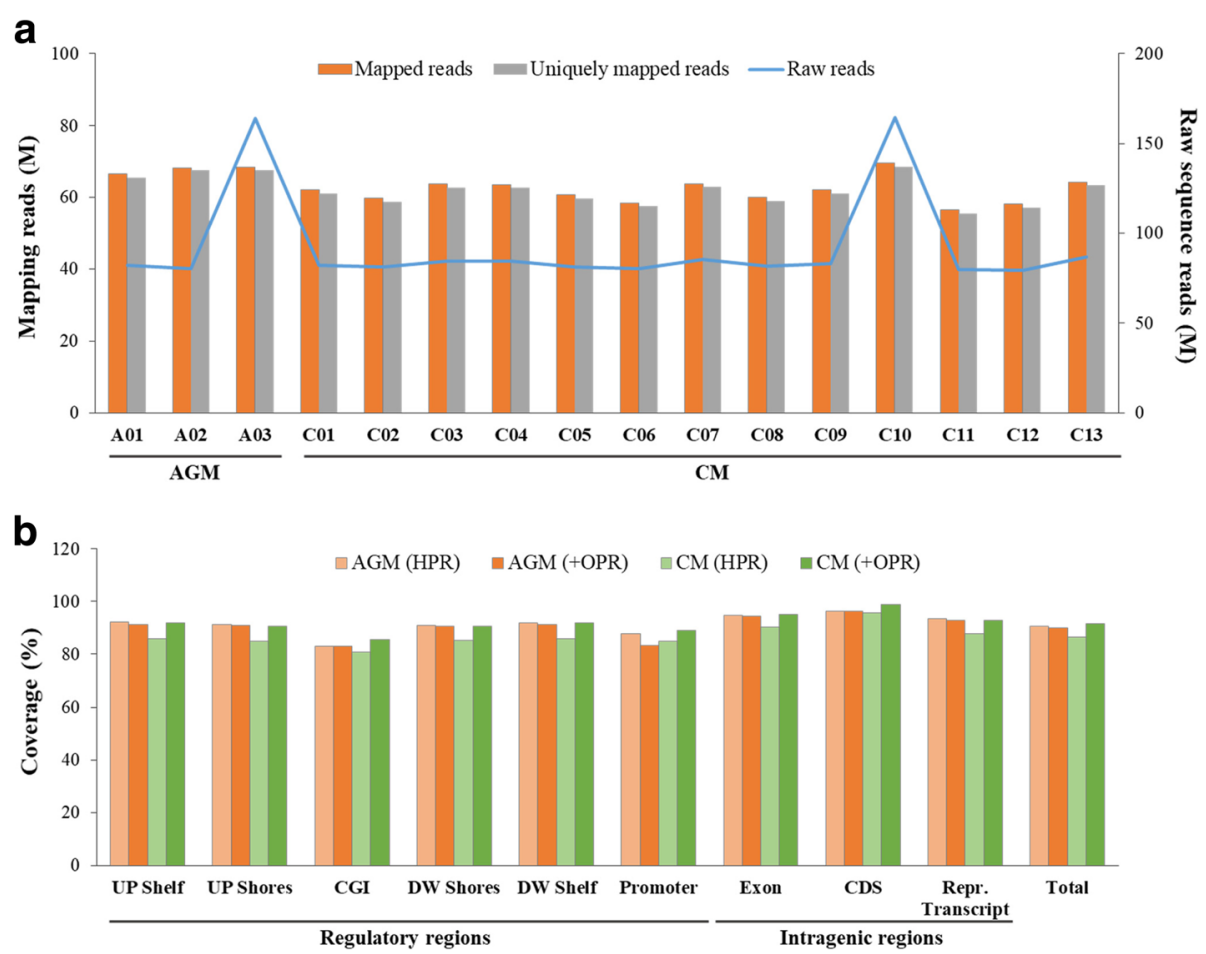

Fig. 4 Read statistics. a Bars show the numbers of mapped reads and uniquely mapped reads, and the line represents the number of raw reads. Each vertical axis shows the number of reads in millions. $\mathbf{b}$ Bars represent the rate of coverage of on-targeted read by genomic regions at a calling depth $\geq$ 5-fold. AGM, African green monkey; CM, cynomolgus macaque; HPR, homologous promoter region; OPR, orthologous promoter region

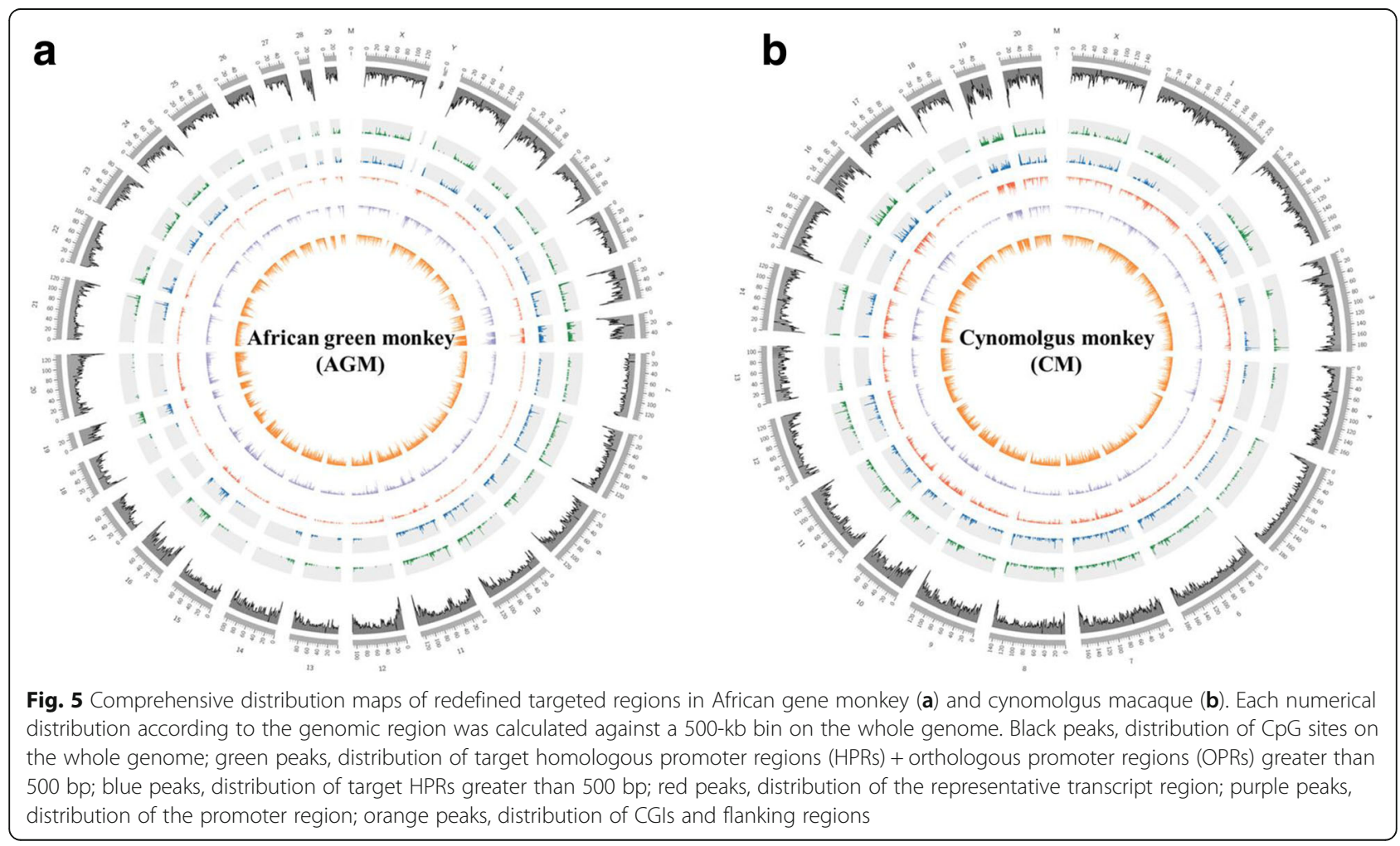



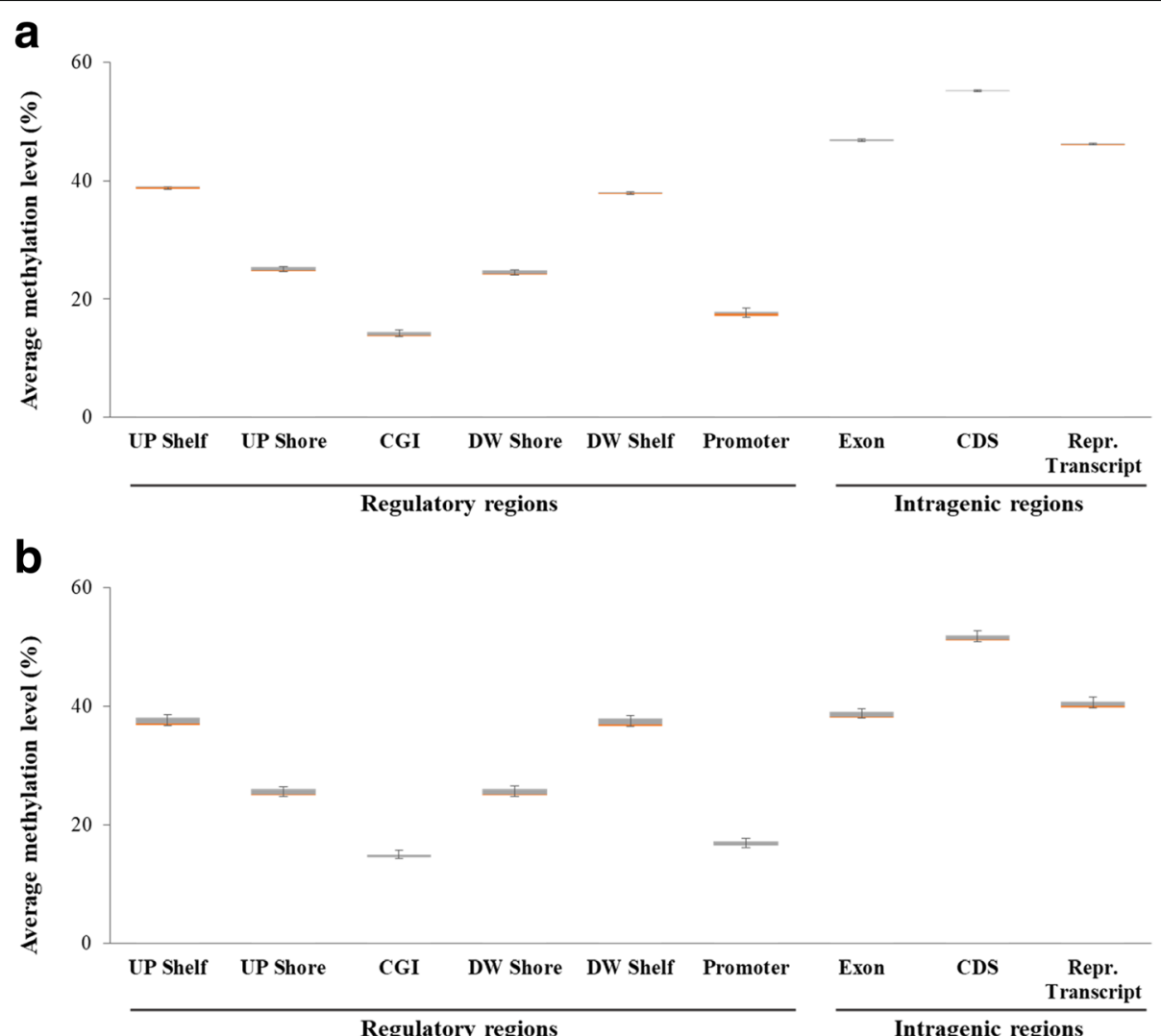

Fig. 6 Average methylation levels according to each genomic region in the African green monkey (a) and cynomolgus macaque (b) genomes. The boxes and error bars indicate the mean and $95 \%$ confidence intervals, respectively

patterns from the CGIs to the outside regions [30]. We confirmed these general global methylation patterns in both the AGM and CM genomic regions (Fig. 6). These results confirmed the reliable performance of the human-based MC-seq method applied to NHP genomes for global methylation analysis.

\section{Discussion}

With the development of microarray hybridization and NGS technologies, researchers must now consider several factors for genome-wide DNA methylation analysis according to the specific research purpose, including the available DNA amount, coverage, resolution, cost, and analysis terms. To facilitate appropriate selection of a genome-wide methylation platform for application to NHP models according to research needs, we classified all of the approaches available into microarray and NGS platforms, and comparatively subdivided the main methods with their details to serve as a guide (Fig. 7 and Table 1).

Although the WGBS method is considered to be the gold standard for genome-wide DNA methylation profiling, it is unsuitable for methylome screening or comparative profiling for diverse applications owing to the high cost and long processing time. The methylated DNA immunoprecipitation (MeDIP) method is easy to apply to any other species, including primates [31], because of the use of methylation-specific antibodies in the DNA enrichment process. However, the critical weak point of MeDIP-chip or MeDIP-seq is the resolution, which hinders methylation quantification at single-nucleotide resolution [32]. Methyl-CpG-binding domain (MBD)-chip or MBD-seq also allows for obtaining broader coverage of the genome, but these methods are also associated with a low resolution problem [33].

To reduce the cost and processing time at the single-base resolution, Infinium $450 \mathrm{~K}$ and $\mathrm{MC}$-seq were suggested as reasonable alternatives to a WGBS platform for clinical DNA methylome studies or epigenome-wide association studies [34]. However, MC-seq appears to be a more attractive alternative platform for methylome analysis at the single-base resolution for large-scale analyses of clinical samples with respect to coverage, technical variation, and concordance of methylation calls [35]. A previous study showed that the Infinium $450 \mathrm{~K}$ array method could 


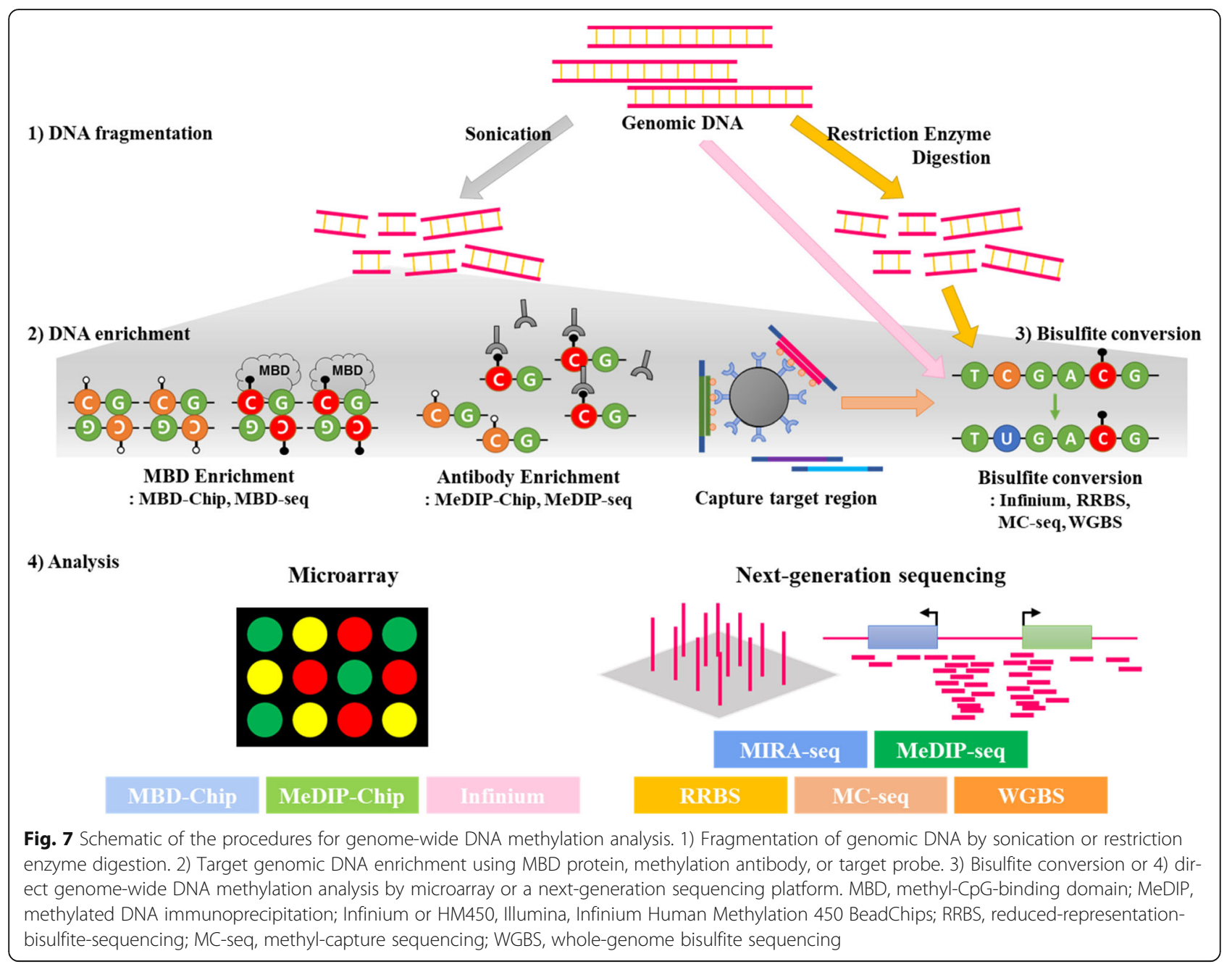

be accurately applied as a cross-species analysis of the DNA methylome of CM muscle tissues [36]. However, the suitability of MC-seq for NHP models has not been assessed to date. Here, we show that the redefined target region provided sufficient resolution ( $\geq 40$-fold), and intermediate wide-coverage ( $\geq 56 \mathrm{Mb}$ coverage) compared with other methylome analysis methods as Infinium $450 \mathrm{~K}$ [36] and WGBS [37]. Thus, we provide the first demonstration that humanbased MC-seq is a practical and valuable approach for analyses of primate models, specifically in AGM and $\mathrm{CM}$.

In this study, the SureSelect human toolkit from Agilent Technologies was used for the target enrichment of the AGM and CM genomes. This toolkit was designed for various target regions, including DNA fragments of CG-rich regions (CGIs, and shore and shelf regions), promoter regions, Refseq genes, Ensembl regulatory features, as well as known cancerand tissue-specific DMRs on the human genome.
Therefore, this MC-seq method is useful for analyses of the methylome. Based on this feature, we expect that our redefined target region might provide basic methylome data from an NHP model. Furthermore, the coverages for the AGM and CM genomes were similar to those obtained with the previous study using the Infinium $450 \mathrm{~K}$ array in CM: the human Infinium $450 \mathrm{~K}$ probes could cover approximately $61 \%$ of the designed regions in the CM genome [36], and the SureSelect human toolkit achieved almost $60 \%$ coverage of the designed regions in the AGM and $\mathrm{CM}$ genomes. Therefore, considering the genomic coverage of MC-seq (1.7-1.8 million CG sites), our results suggest that the SureSelect human toolkit can be applied to methylome analysis for an intermediate genomic range between that obtained with the Infinium $450 \mathrm{~K}$ (298,070 CG sites) [36] and WGBS (21 million CG sites) [37] platforms, applicable for NHP models. Further development and application of human-based MC-seq with NHP models should 
Table 1 Summary of experimental approaches for genome-wide DNA methylation profiling

\begin{tabular}{|c|c|c|c|c|c|c|c|c|}
\hline & ${ }^{\mathrm{a}}$ Species & $\begin{array}{l}\text { bDNA amount } \\
(\mu \mathrm{g})\end{array}$ & Reads & $\begin{array}{l}\text { Genome Coverage } \\
(\mathrm{CpG})\end{array}$ & Influencing factor & Resolution & $\begin{array}{l}\text { Bioinformatics } \\
\text { requirement }\end{array}$ & Cost \\
\hline \multicolumn{9}{|c|}{ Array-based methods ${ }^{d}$} \\
\hline MeDIP-chip & limited & 5 & - & depends on array & $A, B, C, D$ & $\sim 150 \mathrm{bp}$ & ++ & ++ \\
\hline MBD-chip & limited & 5 & - & depends on array & $A, B, C, D$ & $\sim 150 \mathrm{bp}$ & ++ & ++ \\
\hline Infinium & limited & $0.5-1$ & - & $485 \mathrm{~K}$ & $C, E, G$ & Single base & + & + \\
\hline \multicolumn{9}{|c|}{ NGS-based methods ${ }^{e}$} \\
\hline MeDIP-seq & any & $0.3-5$ & $50 \mathrm{M}$ & $\sim 23 \mathrm{M}$ & $A, B, D$ & $\sim 150 \mathrm{bp}$ & +++ & ++ \\
\hline MBD-seq & any & $1-3$ & $30 \mathrm{M}$ & $\sim 23 \mathrm{M}$ & $A, B, D$ & $\sim 150 \mathrm{bp}$ & +++ & ++ \\
\hline RRBS & any & $0.01-2$ & $10 \mathrm{M}$ & $\sim 2 \mathrm{M}$ & $E, F, G$ & Single base & +++ & ++ \\
\hline MC-seq & limited & $1-3$ & $50 \mathrm{M}$ & $3.7 \mathrm{M}$ & $E, G$ & Single base & +++ & ++ \\
\hline WGBS & any & $1-5$ & $>500 \mathrm{M}$ & $>28 \mathrm{M}$ & $E, G$ & Single base & +++++ & +++++ \\
\hline
\end{tabular}

Abbreviations: MeDIP methylated DNA immunoprecipitation, MBD methyl-CpG-binding domain, NGS next-generation sequencing, RRBS reduced-representationbisulfite-sequencing, $M C$-seq methyl-capture sequencing, WGBS whole-genome bisulfite sequencing, $M$ million, $K$ thousand, + very low, ++ low, +++ moderate, ++ ++ high, +++++ very high

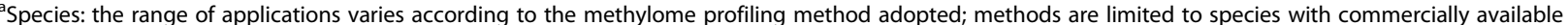
arrays, or species with a complete reference genome available

bNA input varies depending on the protocol

Influencing factors represent the potential sources of genomic region bias. A, CG content; B, CpG density; C, probe hybridization; D, copy number variation; $E_{,}$

bisulfite conversion rate; $F$, enzyme recognition sites; $G$, bisulfite PCR bias

${ }^{d}$ References: MeDIP-Chip [39, 40]; MBD-Chip [40, 41]; Infinium [19, 34, 42-44]

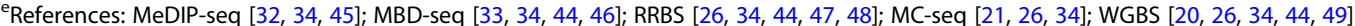

enable reasonable and powerful methylome screening or profiling analyses in various research fields with numerous advantages, including low cost, low bioinformatics requirements, high resolution, negligible interference of influencing factors, high genomic coverage, and requirement of a low sample amount.

\section{Conclusion}

We demonstrated the applicability and accuracy of human-based MC-seq to assay the DNA methylome in blood samples collected from three AGMs and 13 CMs. We adapted the human MC-seq protocol to bisulfite sequencing for NHPs considering inter-species sequence homology and promoter region similarities. The redefined target region provides sufficient resolution (average 47-fold) to analyze the NHP methylome data. Although our method can only make use of $60 \%$ of the human probe-designed target region, it provided genome-wide coverage (1.7-1.8 million CG sites) that is intermediate between that obtained with the Infinium $450 \mathrm{~K}(298,070 \mathrm{CG}$ sites $)$ [36] and WGBS (21 million CG sites) platforms [37]. Humanbased MC-seq has cost, and time effectiveness than WGBS, and has high performance than Infinium $450 \mathrm{~K}$ at the single-base resolution. In the human genome, the targeted probe region includes the cancer- and tissue-specific DMRs, CGIs, Gencode promoters, DMRs or regulatory features in CGIs, shores and shelves, DNase I hypersensitive sites, Refseq genes, and Ensembl regulatory features [38]. Our method can also capture the bisulfite sequences on the NHP genome that target the above-mentioned regulatory regions. Therefore, we conclude that human-based MC-seq can be a suitable approach for DNA methylome profiling of NHP animal models.

\section{Additional files}

Additional file 1: Table S1.The length $(\mathrm{Mb})$ of aligned homologous probe region according to identities and e-value. (DOCX $27 \mathrm{~kb}$ )

Additional file 2: Table S2. CG site distribution according to the genomic region in the redefined target region. (DOCX $28 \mathrm{~kb}$ )

Additional file 3: Table S3. Summary of alignment statistics about sequenced reads. (DOCX $28 \mathrm{~kb}$ )

Additional file 4: Table S4. On-targeted reads and average depth with accumulative depth coverage on CG sites. (DOCX $31 \mathrm{~kb}$ )

Additional file 5: Table S5. Average methylation level on CG sites for each genomic regions (mean \pm s.d.). (DOCX $27 \mathrm{~kb}$ )

\section{Abbreviations}

AGM: African green monkey; CGl: CpG island; CM: Cynomolgus macaque; DMR: Differentially methylated region; HPR: Homologous probe region; MBD: Methyl-CpG-binding domain; MC-seq: Methyl-capture sequencing; MeDIP: Methylated DNA immunoprecipitation; NGS: Next-generation sequencing; OPR: Orthologous promoter region; TSS: Transcription start site; WGBS: Whole-genome bisulfite sequencing

\section{Funding}

This research was supported by Korea Research Institute of Bioscience and Biotechnology (KRIBB) Research Initiative Program grants (KGM4241844 \& KGM4611821), and the Bio \& Medical Technology Development Program of the National Research Foundation funded by the Korean government, MSIP (NRF-2014M3A9B6070243). 


\section{Availability of data and materials}

The data sets supporting the results of this article are included within the manuscript and its additional files. The raw NGS datasets generated during the current study are not publicly available due as analysis is still ongoing, but are available from the corresponding author on reasonable request. Materials: The SureSelect ${ }^{\mathrm{XT}}$ Methyl-Seq Target Enrichment System will be available for purchase from Agilent.

\section{Authors' contributions}

JRL, DSR, and SJP contributed equally to this work. YHK, JWH, JRL, DSR, and SJP designed the project. YHK, and JWH supervised the progress of the project. JRL, DSR, and SJP set up and performed the analyses and contributed to drafting of the manuscript. CSH and HMC prepared blood samples of non-human primates. CSH and HMC generated sequences from the samples. SRL, SUK, YHK, JWH participated in improving the manuscript. All authors read and approved the final manuscript.

\section{Ethics approval}

Ethical approval for collecting blood samples of cynomolgus macaques and African green monkeys was granted by the Institutional Animal Care and Use Committee (KRIBB-AEC-140007, KRIBB-AEC-15031 \& KRIBB-AEC-15046) of the Korea Research Institute of Bioscience and Biotechnology (KRIBB). Animal preparation and study design were conducted according to the Guidelines of the Institutional Animal Care and Use Committee. Blood samples of cynomolgus macaques and African green monkeys were provided by the National Primate Research Center of Republic of Korea.

\section{Competing interests}

The authors declare that they have no competing interests.

\section{Author details}

'Primate Resource Center, Korea Research Institute of Bioscience and Biotechnology, Jeongeup 56216, Republic of Korea. ${ }^{2}$ Theragen Etex Bio Institute, Suwon, Republic of Korea. ${ }^{3}$ National Primate Research Center, Korea Research Institute of Bioscience and Biotechnology, Cheongju 28116, Republic of Korea. ${ }^{4}$ Futuristic Animal Resource and Research Center, Korea Research Institute of Bioscience and Biotechnology, Cheongju 28116, Republic of Korea. ${ }^{5}$ Department of Functional Genomics, KRIBB School of Bioscience, Korea University of Science and Technology (UST), Daejeon 34113 , Republic of Korea.

\section{Received: 30 August 2017 Accepted: 12 April 2018 Published online: 18 April 2018}

\section{References}

1. Carlsson HE, Schapiro SJ, Farah I, Hau J. Use of primates in research: a global overview. Am J Primatol. 2004;63(4):225-37.

2. Fujiyama A, Watanabe H, Toyoda A, Taylor TD, Itoh T, Tsai SF, et al. Construction and analysis of a human-chimpanzee comparative clone map. Science. 2002;295(5552):131-4.

3. Gibbs RA, Rogers J, Katze MG, Bumgarner R, Weinstock GM, Mardis ER, et al. Evolutionary and biomedical insights from the rhesus macaque genome. Science. 2007;316(5822):222-34.

4. Ebeling M, Kung E, See A, Broger C, Steiner G, Berrera M, et al. Genomebased analysis of the nonhuman primate Macaca fascicularis as a model for drug safety assessment. Genome Res. 2011;21(10):1746-56.

5. Huang YS, Ramensky V, Service SK, Jasinska AJ, Jung Y, Choi OW, et al. Sequencing strategies and characterization of 721 vervet monkey genomes for future genetic analyses of medically relevant traits. BMC Biol. 2015;13:41.

6. Lee A, Khiabanian H, Kugelman J, Elliott O, Nagle E, Yu GY, et al. Transcriptome reconstruction and annotation of cynomolgus and African green monkey. BMC Genomics. 2014;15:846.

7. Lankau EW, Turner PV, Mullan RJ, Galland GG. Use of nonhuman primates in research in North America. J Am Assoc Lab Anim Sci. 2014;53(3):278-82.

8. Perelman P, Johnson WE, Roos C, Seuanez HN, Horvath JE, Moreira MA, et al. A molecular phylogeny of living primates. PLoS Genet. 2011;7(3): e1001342.

9. Vallender EJ, Miller GM. Nonhuman primate models in the genomic era: a paradigm shift. ILAR J. 2013;54(2):154-65.
10. Park SJ, Kim YH, Lee SR, Choe SH, Kim MJ, Kim SU, et al. Gain of a new exon by a lineage-specific Alu element-integration event in the BCS1L gene during primate evolution. Mol Cells. 2015;38(11):950-8.

11. Kim YH, Choe SH, Song BS, Park SJ, Kim MJ, Park YH, et al. Macaca specific exon creation event generates a novel ZKSCAN5 transcript. Gene. 2016; 577(2):236-43.

12. Lee JR, Kim YH, Park SJ, Choe SH, Cho HM, Lee SR, et al. Identification of alternative variants and insertion of the novel polymorphic AluYl17 in TSEN54 gene during primate evolution. Int J Genomics. 2016;2016:1679574.

13. Huh JW, Kim YH, Park SJ, Kim DS, Lee SR, Kim KM, et al. Large-scale transcriptome sequencing and gene analyses in the crab-eating macaque (Macaca fascicularis) for biomedical research. BMC Genomics. 2012;13:163.

14. Bird A. The essentials of DNA methylation. Cell. 1992;70(1):5-8.

15. Rakyan VK, Down TA, Thorne NP, Flicek P, Kulesha E, Graf S, et al. An integrated resource for genome-wide identification and analysis of human tissue-specific differentially methylated regions (tDMRs). Genome Res. 2008; 18(9):1518-29.

16. Jones PA. Functions of DNA methylation: islands, start sites, gene bodies and beyond. Nat Rev Genet. 2012;13(7):484-92.

17. Hackett JA, Surani MADNA. Methylation dynamics during the mammalian life cycle. Philos Trans R Soc Lond Ser B Biol Sci. 2013;368(1609):20110328.

18. Ho SM, Johnson A, Tarapore P, Janakiram V, Zhang X, Leung YK. Environmental epigenetics and its implication on disease risk and health outcomes. ILAR J. 2012;53(3-4):289-305.

19. Sandoval J, Heyn H, Moran S, Serra-Musach J, Pujana MA, Bibikova M, et al. Validation of a DNA methylation microarray for 450,000 CpG sites in the human genome. Epigenetics. 2011;6(6):692-702.

20. Ziller MJ, Hansen KD, Meissner A, Aryee MJ. Coverage recommendations for methylation analysis by whole-genome bisulfite sequencing. Nat Methods. 2015;12(3):230-2. 1 p following 2

21. Lee E, Luo J, Wilson JM, Shi H. Analyzing the cancer methylome through targeted bisulfite sequencing. Cancer Left. 2013;340(2):171-8.

22. Rice P, Longden I, Bleasby A. EMBOSS: the European molecular biology open software suite. Trends Genet. 2000;16(6):276-7.

23. Irizarry RA, Ladd-Acosta C, Wen B, Wu Z, Montano C, Onyango P, et al. The human colon cancer methylome shows similar hypo- and hypermethylation at conserved tissue-specific CpG island shores. Nat Genet. 2009:41(2):178-86.

24. Bibikova M, Barnes B, Tsan C, Ho V, Klotzle B, Le JM, et al. High density DNA methylation array with single CpG site resolution. Genomics. 2011;98(4):288-95

25. Kent WJ. BLAT-the BLAST-like alignment tool. Genome Res. 2002;12(4):656-64.

26. Sun Z, Cunningham J, Slager S, Kocher JP. Base resolution methylome profiling: considerations in platform selection, data preprocessing and analysis. Epigenomics. 2015;7(5):813-28.

27. Anupama Khanna AC, Syed F. Epignome methyl-seq kit: a novel postbisulfite conversion library prep method for methylation analysis. Nature Method. 2013;10:3-4.

28. Krueger F, Andrews SR. Bismark: a flexible aligner and methylation caller for Bisulfite-Seq applications. Bioinformatics. 2011;27(11):1571-2.

29. Vinson C, Chatterjee R. CG methylation. Epigenomics. 2012;4(6):655-63.

30. Li E, Zhang Y. DNA methylation in mammals. Cold Spring Harb Perspect Biol. 2014;6(5):a019133.

31. Provencal N, Suderman MJ, Guillemin C, Massart R, Ruggiero A, Wang D, et al. The signature of maternal rearing in the methylome in rhesus macaque prefrontal cortex and T cells. J Neurosci. 2012;32(44):15626-42.

32. Bock C, Tomazou EM, Brinkman AB, Muller F, Simmer F, Gu H, et al. Quantitative comparison of genome-wide DNA methylation mapping technologies. Nat Biotechnol. 2010;28(10):1106-14.

33. Serre $\mathrm{D}$, Lee $\mathrm{BH}$, Ting $\mathrm{AH}$. MBD-isolated genome sequencing provides a high-throughput and comprehensive survey of DNA methylation in the human genome. Nucleic Acids Res. 2010;38(2):391-9.

34. Soto J, Rodriguez-Antolin C, Vallespin E, de Castro Carpeno J, Ibanez de Caceres I. The impact of next-generation sequencing on the DNA methylation-based translational cancer research. Transl Res. 2016;169:1-18.

35. Teh $\mathrm{AL}$, Pan $\mathrm{H}$, Lin $\mathrm{X}$, Lim $\mathrm{Yl}$, Patro CP, Cheong CY, et al. Comparison of methyl-capture sequencing vs. Infinium 450K methylation array for methylome analysis in clinical samples. Epigenetics. 2016;11(1):36-48.

36. Ong ML, Tan PY, Maclsaac JL, Mah SM, Buschdorf JP, Cheong CY, et al. Infinium monkeys: Infinium 450K array for the Cynomolgus macaque (Macaca fascicularis). G3 (Bethesda). 2014;4(7):1227-34. 
37. Mendizabal I, Shi L, Keller TE, Konopka G, Preuss TM, Hsieh TF, et al. Comparative Methylome analyses identify epigenetic regulatory loci of human brain evolution. Mol Biol Evol. 2016;33(11):2947-59.

38. Technologies A. NGS Target enrichment SureSelectXT human methyl-Seq. Agilent Technologies genomics, 5990-9856EN. 2015.

39. Weng YI, Huang TH, Yan PS. Methylated DNA immunoprecipitation and microarray-based analysis: detection of DNA methylation in breast cancer cell lines. Methods Mol Biol. 2009;590:165-76.

40. Nair SS, Coolen MW, Stirzaker C, Song JZ, Statham AL, Strbenac D, et al. Comparison of methyl-DNA immunoprecipitation (MeDIP) and methyl-CpG binding domain (MBD) protein capture for genome-wide DNA methylation analysis reveal CpG sequence coverage bias. Epigenetics. 2011;6(1):34-44.

41. Yegnasubramanian S, Wu Z, Haffner MC, Esopi D, Aryee MJ, Badrinath R, et al. Chromosome-wide mapping of DNA methylation patterns in normal and malignant prostate cells reveals pervasive methylation of geneassociated and conserved intergenic sequences. BMC Genomics. 2011;12:313.

42. Dedeurwaerder S, Defrance M, Calonne E, Denis H, Sotiriou C, Fuks F. Evaluation of the Infinium methylation 450K technology. Epigenomics. 2011; 3(6):771-84.

43. Pan H, Chen L, Dogra S, Teh AL, Tan JH, Lim Yl, et al. Measuring the methylome in clinical samples: improved processing of the Infinium human Methylation450 BeadChip Array. Epigenetics. 2012;7(10):1173-87.

44. Stirzaker C, Taberlay PC, Statham AL, Clark SJ. Mining cancer methylomes: prospects and challenges. Trends Genet. 2014;30(2):75-84.

45. Down TA, Rakyan VK, Turner DJ, Flicek P, Li H, Kulesha E, et al. A Bayesian deconvolution strategy for immunoprecipitation-based DNA methylome analysis. Nat Biotechnol. 2008;26(7):779-85.

46. Lan X, Adams C, Landers M, Dudas M, Krissinger D, Marnellos G, et al. High resolution detection and analysis of $\mathrm{CpG}$ dinucleotides methylation using MBD-Seq technology. PLoS One. 2011;6(7):e22226.

47. Meissner A, Gnirke A, Bell GW, Ramsahoye B, Lander ES, Jaenisch R. Reduced representation bisulfite sequencing for comparative high-resolution DNA methylation analysis. Nucleic Acids Res. 2005:33(18):5868-77.

48. Gu H, Smith ZD, Bock C, Boyle P, Gnirke A, Meissner A. Preparation of reduced representation bisulfite sequencing libraries for genome-scale DNA methylation profiling. Nat Protoc. 2011;6(4):468-81.

49. Lister R, Pelizzola M, Dowen RH, Hawkins RD, Hon G, Tonti-Filippini J, et al. Human DNA methylomes at base resolution show widespread epigenomic differences. Nature. 2009:462(7271):315-22.

\section{Ready to submit your research? Choose BMC and benefit from}

- fast, convenient online submission

- thorough peer review by experienced researchers in your field

- rapid publication on acceptance

- support for research data, including large and complex data types

- gold Open Access which fosters wider collaboration and increased citations - maximum visibility for your research: over $100 \mathrm{M}$ website views per year

At BMC, research is always in progress.

Learn more biomedcentral.com/submissions 